\title{
Complex Multifactorial Genetic Diseases
}

\section{Secondary article}

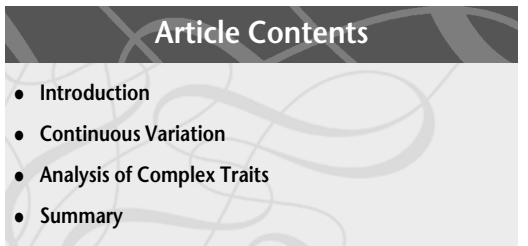

Variation in traits can be due to genetic factors or the environment - nature versus nurture - or to a combination of both. It can be of interest, especially when a trait is detrimental, to model the effect of the genes involved and, beyond that, to map them. Knowledge of disease susceptibility genes may help in the quest for new treatments and cures.

\section{Introduction}

A trait is often of interest by virtue of being either highly desirable, for example intelligence, or highly undesirable, for example cancer. Genetic influence over the trait can be established in many ways including twin studies, clustering statistics and statistical modelling.

Once the existence of a genetic component has been established, the next step is to locate the genes involved. The ease or difficulty of this is influenced by the complexity of the trait and the size of the genetic effect. In multifactorial traits, multiple pathways may lead to both genotypic and phenotypic heterogeneity, the genotypephenotype relationship may be complicated (low or agedependent penetrances), phenocopies may exist, and the phenotype itself may be hard to measure.

\section{Continuous Variation}

The variability exhibited by many traits fails to fit into separate phenotypic classes, but instead forms a spectrum of phenotypes. This is described as continuous variation, and the traits involved are quantitative. A dichotomous trait may have an underlying quantitative trait, with a threshold defining affected status.

In large populations it is often observed that very few individuals possess the extreme phenotypes, but instead progressively more individuals take on values nearer to the population mean. In statistics, the normal distribution (defined by the arithmetic mean and the variance) is often used as an approximation to the distribution of trait values. An additional measurement of interest is the coefficient of variation. This is calculated by dividing the standard deviation by the mean, and is useful for comparing the relative variability of traits when they have different units of measurement.

\section{The role of genes}

The basic difference between traits of this kind and those more qualitative in nature involves the number of genes contributing to the phenotype and the degree to which the phenotype can be modified by environmental factors. Quantitative traits are often governed by many genes, each contributing a small amount to the phenotype and each influenced in a different way by its environment.

A particular gene may be involved in a number of related, or even seemingly unrelated, traits. The myriad phenotypic expressions associated with a single gene are its pleiotropic effects. An example of this is sickle cell anaemia, for which abnormal haemoglobin and hence severe anaemia is the primary effect. Secondary effects include the tendency of the sickle-shaped blood cells to clump together and clog blood vessels in various organs of the body. Heart, kidney, spleen and brain damage are therefore common elements of the syndrome.

\section{Gene-gene interaction}

Gene-gene interaction, or epistasis, occurs when two or more genes catalyse steps in a common pathway. Such interaction can be a source of variability in the phenotype. Whereas dominance involves the masking effect of one allele over the expression of another allele at the same locus, epistasis involves the masking effect of one gene locus on expression at another, usually unlinked, locus. Genetic interaction may also occur if the end products of different pathways each contribute to the same trait. Since proteins produced by genes have various structural or enzymatic functions, this type of interaction is best understood in the context of gene-product interactions.

\section{Gene-environment interaction}

Phenotypic variability will usually be expressed even in a group of organisms that are genetically identical. All such variability is environmental in origin where, in this context, the environment of a gene consists of all physical and chemical factors to which it is exposed. Examples include external factors such as temperature, light and nutrition, and internal factors such as sex, age and availability of substrates, all of which combine to modify the genotypic expression. 
Genotypes may also vary in the degree to which their expression can be modified. The ABO blood groups in humans, for example, show almost no variability irrespective of environment, whereas human intelligence can vary widely with environment.

A phenocopy arises when a response to the environment leads to a phenotype that resembles the effects of a particular gene or group of genes, for example nonfamilial breast cancer. The reverse can also occur, for example when inherited diseases are controlled by drug intervention. An example of this is phenylketonuria, an autosomal recessive disease which can be controlled by diet and drug therapy.

In summary, mutant phenotypes can be induced from genetically normal organisms, and normal phenotypes can be induced in genetically mutant organisms by the effects of environment. Thus, an understanding of gene-environment interaction aids in predicting disease rates and provides a basis for well-informed recommendations for disease prevention.

\section{Models of gene-environment interaction}

Ottman (1990) proposed five models of gene-environment interaction under various assumptions. To discriminate among these five models, individuals are classified according to presence or absence of predisposing genetic and environmental risk factors. Although environmental information may be easy to establish, determining the presence of genetic risk factors can be more problematic. Usually these have not been fully identified, so family history data are used. There is potential for significant misclassification by this approach, however, since the probability of detecting evidence of a positive family history increases with the number of first-degree relatives.

\section{Estimating genetic involvement}

\section{Components of variance}

The study of quantitative traits suggests that total (phenotypic) variance can be modelled as the sum of three components: environmental variance, genetic variance, and variance due to gene-environment interaction. Mathematically,

$$
V_{\mathrm{P}}=V_{\mathrm{G}}+V_{\mathrm{E}}+V_{\mathrm{GE}}
$$

The broad definition of heritability, or the proportion of phenotypic variance attributable to total genetic factors, is expressed as:

$$
H^{2}=V_{\mathrm{G}} / V_{\mathrm{P}}
$$

In fact, $V_{\mathrm{G}}$ can be broken down further, to additive, dominant and epistatic effects. If these are expressed as $V_{\mathrm{A}}$, $V_{\mathrm{D}}$, and $V_{\mathrm{I}}$ respectively,

$$
V_{\mathrm{G}}=V_{\mathrm{A}}+V_{\mathrm{D}}+V_{\mathrm{I}}
$$

The more commonly used, narrow, definition of heritability is then expressed as:

$$
h^{2}=V_{\mathrm{A}} / V_{\mathrm{P}}
$$

The values of these variance components can be estimated by observing trait correlation in relatives, for example using the LISREL program (Joreskog and Sorbom, 1986), which uses stringent maximum likelihood techniques to fit genetic covariance structure models to phenotypic data.

\section{Twin studies}

A widely used method in human population genetics for estimating the relative contributions of genes and environment to interindividual trait variability is the twin study. It offers a unique opportunity to distinguish between the influences of shared genes and shared environment by comparing the resemblance between monozygotic (MZ) and dizygotic (DZ) twins for a particular trait in order to obtain an estimate of its heritability. MZ twins share $100 \%$ of their genetic material, whereas DZ twins share on average $50 \%$, as do siblings. Greater resemblance of $\mathrm{MZ}$ than DZ twins for a particular trait is thus an indication of the importance of genetic factors.

For continuously distributed traits, such as blood pressure or cholesterol levels, an estimate of heritability is given by the formula:

$$
h^{2}=2\left(r_{\mathrm{MZ}}-r_{\mathrm{DZ}}\right)
$$

where $r_{\mathrm{MZ}}$ and $r_{\mathrm{DZ}}$ are the intra-class correlations for $\mathrm{MZ}$ and DZ twins respectively. Values of $r_{\mathrm{MZ}}$ and $r_{\mathrm{DZ}}$, such that $r_{\mathrm{MZ}}<r_{\mathrm{DZ}}$, indicate the importance of shared environment, the magnitude of which may be estimated by:

$$
c^{2}=2 r_{\mathrm{DZ}}-r_{\mathrm{MZ}}
$$

There are certain limitations of twin studies. They do not test any explicit model for individual differences and do not consider nongenetic transmission. Additionally, the classical approach of comparing $\mathrm{MZ}$ and $\mathrm{DZ}$ correlation ignores information available in variances and covariances, which is of importance in the genetic analysis of sex and generational differences. Furthermore, it is sometimes difficult to confirm the monozygotic origin of twins, and their in utero environments, although similar, are not necessarily identical. The latter effect means that environmentally induced differences may exist even before birth and, later, somatic mutations in their postnatal development may lead to genetic differences in selected cells. Nevertheless, whenever breeding experiments are not possible, as in the case of humans, twin studies provide the best means of assessing what proportion of variability amongst individuals is due to heritable differences and what is due to environmental differences. 


\section{Analysis of Complex Traits}

\section{What are complex traits?}

Traits can be complex at many different levels. Genetically, traits can be classified into three categories: monogenic, where a single gene determines disease expression; oligogenic, where a major gene exists together with one or more modifier genes; and polygenic, where many genes of small effect exist and complex interactions occur. In terms of this classification, polygenic traits are the most complex, although oligogenic traits, and even monogenic traits, can be complicated by factors such as locus heterogeneity.

\section{Genetic epidemiology}

Twin studies are commonly performed to investigate genetic involvement in a trait. However, a disease may be genetic without necessarily being familial, for example when somatic mutations occur. It is therefore important to illustrate familiality by examining the clustering of the trait within families. This is carried out using the parameter 'relative risk', $\lambda_{\mathrm{R}}$, which calculates the increased risk of disease to a relative $(\mathrm{R})$ of an affected individual over the population risk. Most commonly, this is quoted for sibs, $\lambda_{\mathrm{s}}$. Generally, the greater the value of $\lambda_{\mathrm{S}}$, the easier the genetic mapping of the disease. Segregation analysis can then be used to assess evidence for a major gene, and multilocus involvement can be assessed by the examination of $\lambda_{\mathrm{R}}$ for various relative types (Risch, 1990).

Whenever multiple genes play a role, the parameter $\lambda_{\mathrm{S} ; \text { gene }}$ indicates the degree of disease clustering attributable to that particular gene and is calculated by:

$$
\lambda_{\mathrm{S} ; \text { gene }}=\mathrm{e}^{\pi \ln }
$$

where $\pi$ is the proportion of disease attributable to the

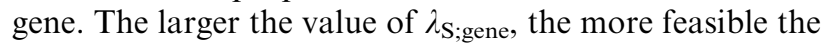
genetic mapping of it becomes.

\section{Heterogeneity}

Various genes, or groups of genes, may lead to the same phenotypic expression. This phenomenon is called locus heterogeneity and is especially common in polygenic traits. It can also occur in simpler traits, for example in breast cancer where at least two rare mendelian forms of the disease exist. On a different level, allelic heterogeneity exists when various alleles (or haplotypes) in a single gene lead to the same phenotypic expression. Isolated populations are more homogeneous both genetically and phenotypically, so they are often chosen for mapping studies. It is debatable, however, whether or how results from these can be generalized to other outbred populations.

\section{Complex phenotypes}

Increased complexity can arise through the phenotype itself, due to low penetrance, phenocopies and other environmental interactions. Some phenotypes are difficult to measure, and it may not be clear what the true trait of interest is. This is the case for psychiatric illnesses where diagnosis is confounded by variability in, and perception of, symptoms over time. In such cases, multiple phenotypes are often analysed, and then corrections must be made for multiple testing.

\section{Analysis methods}

Many methods are available for the analysis of complex traits in humans. These can be categorized into three main approaches: classical linkage analysis, allele-sharing linkage analysis and association analysis. Details of software for carrying out these methods are available at http:// linkage.rockefeller.edu/.

\section{Genome-wide versus candidate region}

Until recently, genetic studies were generally performed on candidate regions. These are regions containing a gene, or gene family, whose function suggests its involvement in the trait. In 1994 the first genome-wide search for genes was published for type 1 diabetes (Davies et al., 1994). It involved a systematic analysis of genetic markers across the entire genome. Statistically, the difference between these two approaches is the critical value used to establish significant evidence for linkage, and it reflects the number of markers analysed.

Significance levels are important as they directly affect the false-positive rate and the power. The power of an analysis is of paramount importance because it indicates the probability of correctly rejecting $H_{0}$. A well designed analysis is one with a low false-positive rate and high power. In genome-wide linkage, $p$ values of $4.9 \times 10^{-5}$ and $2.2 \times 10^{-5}$ have been suggested for significance at the $5 \%$ level in classical and allele-sharing analyses, respectively (Kruglyak and Lander, 1995). A $p$ value of $5 \times 10^{-8}$ has been proposed for genome-wide association studies (Risch and Merikangas, 1996), although this may be amended as more abundant information on linkage disequilibrium across the genome becomes available. Where it is not clear what the critical value should be, simulations can be performed to estimate reasonable thresholds.

\section{Classical linkage analysis}

Linkage occurs when two loci, for example a marker and a disease mutation, lie close together on the same chromosome, and hence they cosegregate. In this context, closeness is measured by the recombination fraction, $\theta$, the probability of a recombination event between the loci. A recombination fraction of 0.5 indicates that loci are unlinked or, in other words, segregating independently as if 
on different chromosomes. A lower recombination fraction indicates the presence of linkage.

Classical linkage analysis is parametric in the sense that it is necessary to specify an inheritance model for the disease. This is given by the allele frequencies and the penetrance functions. Evidence for linkage is assessed via the maximum lod score (logarithm of odds of departure of actual results from expected), , maximized over $\theta$, and calculated as follows:

$$
\hat{Z}=\log _{10}\left\{\frac{P\left(\text { data } \mid H_{1}: \theta=\hat{\theta}\right)}{P\left(\text { data } \mid H_{0}: \theta=0.5\right)}\right\}
$$

A genetic map consisting of 300 polymorphic markers (resolution of approximately $5-10 \mathrm{cM}$ ) is commonly used for genome-wide search. For such an analysis $=3.3$ indicates significant evidence for linkage with a falsepositive rate of $5 \%$, equivalent to a $p$ value of $4.9 \times 10^{-5}$. Much computer software is available to calculate the lod score, for example LINKAGE (Lathrop et al., 1984). Exact computation can be too intensive for some problems, for example if marriage loops or inbreeding occur, and in these cases Markov chain Monte Carlo methods, such as the Gibbs sampler, can be used as alternatives.

If penetrance is unknown, an affecteds-only analysis can be performed, in which all unaffected individuals are considered to have an unknown phenotype. A heterogeneity parameter, estimating the proportion of families linked, can also be maximized along with $\theta$. If some feature of the heterogeneity is known, sequential analysis may be performed. This involves analysing families in an order that has been determined independent of genotype information, for example by age at onset. These methods can be applied to both qualitative and quantitative traits.

Classical linkage analysis is powerful when the correct inheritance model is used, for example in monogenic diseases where segregation analyses can provide reasonable parameter estimates. For more complex traits, performing two analyses, one under a general dominant and one under a general recessive model, generates reasonable power. This method, implemented on extended pedigrees, was successful in locating the disease genes $B R C A 1$ and $B R C A 2$, implicated in breast cancer.

\section{Allele-sharing linkage analysis}

Allele-sharing methods are nonparametric in the sense that a disease model need not be specified. These methods can be implemented using any type of affected relative pair, but mostly affected sib pairs (ASPs) are used. The idea is to compare the inheritance pattern in the ASP with that expected by mendelian inheritance. Under mendelian laws, a sib pair will share 2,1 or 0 alleles identical by descent (IBD) $25 \%, 50 \%$ and $25 \%$ of the time, respectively, where two alleles are said to be IBD if both are copies of the same ancestral allele. The alternative hypothesis is based on the fact that, if linkage exists, there should be an excess of ASPs sharing two alleles IBD. When IBD status can be determined unambiguously, the $\chi^{2}$ test is used ( $2 \mathrm{df}$ ). Alternatively, the IBD status of paternal and maternal alleles can be assessed independently, using two $\chi^{2}$ tests ( 1 df). This allows paternal versus maternal inheritance to be determined.

Usually IBD status cannot be determined unambiguously and the test becomes one of maximum likelihood over the possible IBD states. Evidence for linkage can then be quantified using a lod score. For genome-wide ASP linkage analysis, $Z=3.6$, equivalent to a $p$ value of $2.2 \times 10^{-5}$, provides significant evidence for linkage at the $5 \%$ level. Highly polymorphic markers increase the chance of being able to distinguish all four parental markers, and approximately 300 of these are needed to cover the genome. If no parental information is available, for example in late-onset diseases such as rheumatoid arthritis, the test can be performed using identity by state (IBS) information, that is using alleles of the same type but not necessarily the same ancestral origin. This method, however, is much less powerful.

As in classical linkage analysis, a heterogeneity parameter can be added to ASP analysis. This, however, is less useful here because it becomes increasingly difficult to distinguish linkage from noise when smaller families are used. Extensions to ASP analysis have also been developed for quantitative traits. This was first proposed by Haseman and Elston (1972) using least-squares regression of the squared trait difference in sibs and the number of IBD alleles shared. Other methods also exist based on trait differences in sibs. Extreme discordant or concordant sib pairs have been proposed as the most powerful sibship type for these kinds of analyses.

Allele-sharing methods can be more powerful than classical linkage analyses if family structures are small or if model specification is problematic. In complex diseases where ASP families are more easily ascertained than extended pedigrees, an allele-sharing method is often preferable for detecting linkage. ASP analysis was used in the genome-wide search for type 1 diabetes susceptibility genes, and was successful in confirming the importance of the human leucocyte antigen (HLA) region, and the identification of other novel loci.

\section{Association studies}

Association studies were originally the method of choice for implicating a locus in a disease. As markers became more abundant and informative, linkage studies became preferable. The development of new association-based tests, however, has reawakened interest in these methods.

The basic concept of association is that disease occurs with the presence of a particular allele at a marker locus. This is assumed to be due to linkage disequilibrium, $\delta$, between a disease allele and a marker allele. This occurs 
when they appear on the same haplotype more often than is expected. Biallelic markers such as single nucleotide polymorphisms (SNPs) are preferable in such analyses. This is because association tests are allele specific, and, for biallelic markers, no a priori hypotheses regarding diseaseassociated alleles or multiple testing corrections need to be made. A $\chi^{2}$ test is carried out to compare genotype status (usually carriage of a particular allele) between cases and controls. An odds ratio can also be calculated to estimate the risk of disease to an individual carrying the particular allele. Alternatively, a $2 \times 3$ contingency table can be constructed by genotype. Genotypic relative risks can then be calculated to determine whether there is increased risk to heterozygote or homozygote carriers of the allele of interest compared with that in noncarriers. This also gives information on the mode of inheritance of the disease at that locus.

One disadvantage of these methods is that spurious results can occur if case and control populations are badly matched, or if admixture confounds either population. It is also not currently possible to account for either locus or allelic heterogeneity, especially the latter since these methods are allele specific.

More recently, the transmission disequilibrium test (TDT) has been introduced (Spielman et al., 1993). It is a $\mathrm{McNemar}$ test, with a composite null hypothesis equivalent to $\delta(2 \theta-1)=0$, and tests for biased transmission from heterozygous parents to affected offspring. In the presence of both linkage and association, transmission will be biased in favour of the disease-associated allele. This test has the increased sensitivity of association tests and is robust to population admixture. It is not, however, robust to either locus or allelic heterogeneity. The TDT was used successfully to implicate the insulin gene region in type 1 diabetes.

The TDT and other association tests are based on detecting $\delta \neq 0$, and since linkage disequilibrium extends over very small distances (much less than $1 \mathrm{cM}$ in most populations), these tests have been used mainly in candidate region analyses. However, with a map consisting of $500000 \mathrm{SNPs}$, and imposing a significance threshold of $p=5 \times 10^{-8}$, it has been suggested (Risch and Merikangas, 1996) that genome-wide TDT analyses may be not only plausible, but a superior alternative to ASP linkage analysis for locating small-effect genes. Such a dense map is now an objective of the Human Genome Project. In the future we may therefore see the rise of association-based studies as a method for genome-wide search.

\section{Summary}

Complex multifactorial traits are, as their name would imply, complicated in nature. The methodologies dis- cussed here for determining genetic involvement in traits and the mapping of genes only scrape the surface of a vast and dynamic scientific field. There is no panacea for the genetic mapping of complex traits, and each disease has unique complexities to be addressed. New methodologies are constantly being developed as technologies change. The dawn of complete human genome sequence will undoubtedly bring with it new opportunities for the analysis of complex traits.

\section{References}

Davies JL, Kawaguchi Y, Bennett ST et al. (1994) A genome-wide search for human type 1 diabetes susceptibility genes. Nature 371: 130-136.

Haseman JK and Elston RC (1972) The investigation of linkage between a quantitative trait and a marker locus. Behavioral Genetics 2: 3-19.

Joreskog KG and Sorbom D (1986) LISREL: Analysis of Linear Structural Relationships by the Method of Maximum Likelihood. Chicago, Illinois: National Educational Resources.

Lander E and Kruglyak L (1995) Genetic dissection of complex traits: guidelines for interpreting and reporting linkage results. Nature Genetics 11: 241-247.

Laboratory of Statistical Genetics Rockefeller University (1996) Web Resources of Genetic Linkage Analysis. [http://linkage.rockefeller.edu/ ].

Lathrop GM, Lalouel JM, Julier C and Ott J (1984) Strategies for multilocus linkage analysis in humans. Proceedings of the National Academy of Sciences of the USA 81: 3443-3446.

Ottman R (1990) An epidemiologic approach to gene-environment interaction. Genetic Epidemiology 7: 177-185.

Risch N (1990) Linkage strategies for genetically complex traits. I. Multilocus models. American Journal of Human Genetics 46: 222-228.

Risch N and Merikangas K (1996) The future of genetic studies of complex human diseases. Science 273: 1516-1517.

Spielman RS, McGinnis RE and Ewens WJ (1993) Transmission test for linkage disequilibrium: the insulin gene region and insulin-dependent diabetes mellitus (IDDM). American Journal of Human Genetics $\mathbf{5 2}$ $506-516$.

\section{Further Reading}

Falconer DS (1983) Introduction to Quantitative Genetics, 2nd edn. New York: Longman.

Khoury MJ, Beaty TH and Cohen BH (1993) Fundamentals of Genetic Epidemiology. New York: Oxford University Press.

Kirkwood BS (1988) Essentials of Medical Statistics. Oxford: Blackwell Scientific.

Lander ES and Schork NJ (1994) Genetic dissection of complex traits. Science 265: 2037-2047.

Neale MC and Cardon LR (1992) Methodology for Genetic Studies of Twins and Families. Dordrecht: Kluwer Academic.

Ott J (1991) Analysis of Human Genetic Linkage. Baltimore, MD: Johns Hopkins University Press.

Pawlowitzki I-H, Edwards JH and Thompson EA (eds) (1997) Genetic Mapping of Disease Genes. London: Academic Press.

Schaid DJ (1998) Transmission disequilibrium, family controls, and great expectations. American Journal of Human Genetics 63: 935-941.

Weeks DE and Lathrop GM (1995) Polygenic disease: methods for mapping complex disease traits. Trends in Genetics 11: 513-519. 